\title{
Efecto del entrenamiento pliométrico en el control neuromuscular y la flexibilidad de miembro inferior en el equipo de fútbol masculino Cosdecol
}

\section{Plyometric training effect on neuromuscular control and lower limb flexibility in the Cosdecol men's soccer team}

\author{
Felipe Monsalve-Vélez ${ }^{1}$ iD; Sara Betancur-Henao ${ }^{\text {iD }}$; Daniela Buriticá-Ochoa ${ }^{1}$; ; Daniela

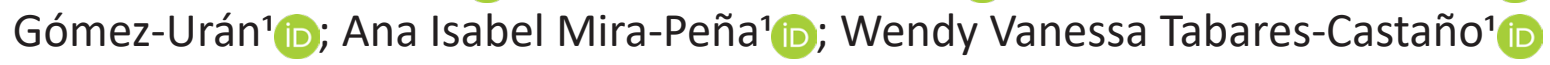

'Universidad CES. Medellín - Antioquia, Colombia; fmonsalvev@ces.edu.co; betancurh.sara@uces.edu.co; buritica.daniela@uces.edu.co; gomezu.daniela@uces.edu.co; mira.ana@uces.edu.co; tabares.wendy@uces.edu.co

Cómo citar: Monsalve-Vélez, F.; Betancur-Henao, S.; Burutucá-Ochoa, D.; Gómez-Uran, D.; Mira-Peña, A.l.; Tabares-Castaño, W.V. 2021. Efecto del entrenamiento pliométrico en el control neuromuscular y la flexibilidad de miembro inferior en el equipo de fútbol masculino Cosdecol. Rev. Digit. Act. Fis. Deport. 7(1):e1529. http://doi.org/10.31910/rdafd.v7.n1.2021.1529

Artículo de acceso abierto publicado por Revista Digital: Actividad Física y Deporte, bajo una licencia Creative Commons CC BY-NC 4.0

Publicación oficial de la Universidad de Ciencias Aplicadas y Ambientales U.D.C.A, Institución de Educación Superior Acreditada de Alta Calidad por el Ministerio de Educación Nacional.

Recibido: Abril 24 de 2020 Aceptado: Octubre 29 de 2020 Editado por: Álvaro José Gracia Díaz

\section{RESUMEN}

Introducción: A nivel mundial, el fútbol es el deporte más practicado, que evidencia un mayor índice de lesiones, especialmente, de miembros inferiores, debido a que la velocidad, la potencia, los saltos y los cambios de dirección durante el juego, obligan a absorber cargas, teniendo una relación directa con los déficit en el control neuromuscular; dicha alteración ha sido identificada como factor de riesgo de lesiones para las extremidades, que es modificable mediante el entrenamiento y puede mejorar el rendimiento deportivo. Objetivo general: Determinar el efecto del entrenamiento pliométrico en el control neuromuscular y la flexibilidad de la extremidad inferior, en futbolistas de un club deportivo de fútbol. Métodos: Estudio cuantitativo, ensayo clínico con diseño correlacional de tipo longitudinal prospectivo. Se desarrolló un protocolo de intervención de 6 semanas de duración, donde se incluyeron 14 jugadores de fútbol, se implementó un cuestionario diligenciado por cada participante y se efectuó una batería de pruebas físicas antes y después del protocolo. Al finalizar, se realizó el análisis estadístico, con el paquete estadístico SPSS V21. Resultados: Se encontró una edad promedio de 18,29 $\pm 1,86$ años; respecto a los antecedentes de lesión, se encontró lesión ligamentosa (42.9\%), lesión muscular $(21,4 \%)$, lesión ósea (14,3\%), lesión tendinosa $(7,1 \%)$ y el $14,3 \%$, no reportó antecedentes. Con relación a la flexibilidad evaluada con test de Bleck y Ely, posterior a la intervención de 6 semanas, todos los deportistas mostraron cambios importantes en la elongación de los flexores y extensores de rodilla. Respecto al control neuromuscular evaluado con el SEBT, se evidenciaron cambios importantes en las distancias alcanzadas, predominantemente, en las direcciones posteromedial, medial y posterolateral. En el test de salto, se identificó cambios importantes en el comparativo antes y después. Conclusión: 
Tras la aplicación de un entrenamiento pliométrico, adicional al convencional, se pueden evidenciar cambios positivos en la flexibilidad de miembros inferiores, específicamente, en la musculatura, que interviene en la flexión y la extensión de rodilla. Igualmente, al conocer las implicaciones neuromusculares para la realización de la actividad deportiva, se resalta la importancia de la inclusión del entrenamiento pliométrico, porque logra la activación adecuada y pertinente de todos los componentes neuromusculares, disminuyendo alteraciones en miembros inferiores, que predisponen a sufrir alguna lesión en dicho segmento.

Palabras clave: Control neuromuscular; Flexibilidad; Pliometría; Fútbol; Extremidad inferior.

\section{ABSTRACT}

Introduction: Globally, football is the most practiced sport that evidences a higher rate of injuries, especially of the lower limbs, due to the fact that speed, power, jumps and changes of direction during the game force to absorb burdens, with a direct relationship with deficits in neuromuscular control; this alteration has been identified as a risk factor for injuries to the extremities, which can be modified through training and improving sports performance. Objective: Determine the effect of plyometric training on neuromuscular control and lower extremity flexibility in football players of a football club. Methods: Quantitative study, clinical trial with correlational design of longitudinal prospective type. A 6-week intervention protocol was developed, including 14 football players, a questionnaire filled out by each participant was implemented and a battery of physical tests was performed before and after the protocol. At the end, the statistical analysis was carried out with the statistical package SPSS V21. Outgrowth: Was found an average age of $18.29 \pm 1.86$ years, with respect to the antecedents of injury, was found ligamentous injury (42.9\%), muscle injury (21.4\%), bone injury (14.3\%), tendon injury (7.1\%) and $14.3 \%$ reported no history. In relation to the flexibility evaluated with Bleck and Ely tests, after the 6-week intervention, all the sportsmen showed important changes in the elongation of the knee flexors and extensors. Respect to the neuromuscular control evaluated with the SEBT, important changes in the distances reached were evidenced, predominantly in the posteromedial, medial and posterolateral directions. The jump test identified important changes in the comparison before and after. Conclusion: After applying the plyometric training protocol, positive changes in the elongation of knee flexors can be evidenced mainly. It is also clearly beneficial for sports performance and injury prevention, as it achieves optimal activation of all the neuromuscular components of the lower limb involved in the technical gesture.

Keywords: Neuromuscular control; Flexibility; Plyometric; Soccer; Lower limb.

\section{INTRODUCCIÓN}

El fútbol es la modalidad deportiva más popular y practicada en el mundo. Según el conteo de practicantes realizado en el último gran censo publicado por la FIFA, en el 2006 (Kunz, 2006), a nivel mundial, se estima que el $4 \%$ de la población mundial participa activamente de la práctica del fútbol. La velocidad, la potencia, los saltos y los exigentes cambios de dirección durante el juego, obligan a absorber cargas de alto impacto, especialmente, en la pelvis, las rodillas y los pies, generando lesiones de predominio en las extremidades inferiores. La incidencia general de estas lesiones, asociadas a la práctica en el fútbol, es de 0,5 a 9 lesiones por cada 1.000 horas de actividad del jugador, que varía durante las competencias, con registros de 10,2 a 42,5 por cada 1.000 horas de juego (Llana et al. 2010).

Hawkins \& Fuller (1999) encontraron que el $41 \%$ de todas las lesiones fueron causadas por contacto físico, mientras que $59 \%$ no incluyeron en su mecanismo de lesión algún contacto, sino que fueron causadas por factores intrínsecos a la práctica deportiva y las condiciones del jugador (Hawkins \& Fuller, 1999). Las lesiones pueden conducir, tanto a ausentismos en la práctica deportiva como al retiro definitivo de la misma, según la complejidad de la lesión, generando una posible disminución del rendimiento físico, deterioro en el trabajo del equipo en general y los altos costos para el sistema de salud.

Los deportes de alta competición, se caracterizan por padecer una mayor incidencia, donde se involucran múltiples factores neuromusculares implicados en el ciclo de estiramiento-acortamiento; sin embargo, no existe claridad sobre el direccionamiento de este trabajo en los protocolos de entrenamiento. Dicha alteración en el control neuromuscular en la extremidad inferior ha sido identificada como factor 
de riesgo de lesión para este segmento corporal en específico y ha recibido especial atención en los últimos años, debido a que las diferencias entre el género, patrones de movimiento y activación muscular se relacionan, probablemente, con un mayor riesgo (Lephart . 1998). Se ha demostrado que los factores de riesgo de lesiones asociadas a la variación del control neuromuscular son modificables, a través del entrenamiento, lo que conduce a un mejor rendimiento deportivo; no obstante, el éxito de los programas de prevención de lesiones que incorporan aspectos del entrenamiento neuromuscular son variables y poco congruentes (Cardona \& Osorio, 2008). Por lo anterior, será necesario ampliar el conocimiento técnico y clínico enfocado al deporte más popular del mundo, que está en proceso de transformación, desde el direccionamiento físico, que se les da a los participantes, el aumento en los requerimientos corporales y las nuevas posibilidades de intervención que potencializan la práctica.

Es por ello, que el presente proyecto de investigación pretende determinar el efecto del entrenamiento pliométrico en el control neuromuscular y la flexibilidad de la extremidad inferior en futbolistas del Club Deportivo COSDECOL, de la categoría primera B. Se pretende, con los resultados del presente estudio, generar un impacto positivo en la gran cantidad de deportistas que practican fútbol, a nivel institucional, local y regional; además, se busca tener implicaciones positivas en la salud pública, porque con el mismo, se podrá dar a conocer una nueva perspectiva acerca del entrenamiento y direccionamiento técnico, relacionado con las demandas y las características biomecánicas que involucra este deporte, dando así, nuevas herramientas que faciliten la prevención de lesiones derivadas de la práctica deportiva, disminución de la incidencia de las mismas, reducción de costos para el sistema de salud y un aumento en el rendimiento físico del practicante.

\section{MATERIALES Y MÉTODOS}

Este estudio tiene un enfoque cuantitativo, con diseño correlacional, prospectivo. La población incluyó a 31 jugadores del equipo de fútbol masculino COSDECOL, quienes cumplieron con los siguientes criterios de inclusión: aceptar voluntariamente la participación en el estudio, entrenar mínimo 2 veces por semana con el equipo de fútbol y llevar practicando fútbol, en categoría competitiva, por al menos un año. Los participantes fueron sometidos a un protocolo de intervención de 6 semanas, con dos sesiones de entrenamiento por semana. Para ser incluidos en los análisis finales, cada sujeto debió tener que completar, el $80 \%$ de todas las sesiones de intervención (es decir, 8 de 12 sesiones) y tuvo que participar en ambas evaluaciones (inicial y final). Finalmente, 14 deportistas cumplieron con todos los requerimientos mencionados previamente.

Para el análisis de los datos de las variables que corresponden a las características sociodemográficas y antropométricas, se verificó la distribución de los mismos, donde las variables: 1) Edad; 2) Peso; 3) Estatura y 4) Índice de masa corporal, se comportan de manera normal, por lo tanto, para analizar los datos se utilizó la media con desviación estándar. Los datos de lugar de residencia, se analizaron con frecuencia y porcentaje.

Asimismo, para las analizar las características del entrenamiento las variables de tipo de: 1) Lesión deportiva; 2) Superficie de entrenamiento; 3) El uso de estabilizadores articulares externos; 4) Posición de juego y 5) La dominancia, se representan por tabla de frecuencias y porcentajes. La variable frecuencia de entrenamiento se comportó de manera no normal, por lo tanto, se utilizó la mediana con rango intercuartil, para analizarla. Asimismo, el tiempo en la práctica deportiva, se comportó de manera normal, por esto, se analizó con media acompañada de desviación estándar.

Finalmente, para el análisis de variables clínicas, con el objetivo de describir la medición de la flexibilidad, con el test de Bleck, en la primera toma, se analizaron los datos con frecuencias y porcentajes, tomando la variable como cualitativa. En adición a lo anterior y dado que los datos obtenidos en la medición del Test de Bleck del miembro inferior derecho e izquierdo antes de la intervención se distribuyen de manera normal y la medición después de la intervención distribuye no normal, se decidió aplicar la prueba Wilcoxon, debido a que la prueba $T$ student puede perder precisión en distribución no paramétrica, lo mismo se aplicó, para el índice de simetría, obtenido a través del hop test, porque la medición antes, se distribuyó de manera no normal y la medición después, normal. Las pruebas que se aplicaron para SEBT fueron Wilcoxon para todas las direcciones, a excepción de la lateral y posterior derecho, donde se utilizó la prueba T-student. 


\section{RESULTADOS}

Características sociodemográficas y antropométricas. Respecto a la edad, se obtuvo una media de $18,29 \pm 1,86$ años; asimismo, la media del peso fue $65,71 \pm 8$ kilogramos; con relación a la estatura, se encontró un promedio de 1,74 $\pm 0,05$ metros; el índice de masa corporal presentó una media de $21,42 \pm 2,06 \mathrm{Kg} / \mathrm{m} 2$, estos valores están dentro del intervalo normal, según la clasificación dada por la OMS $(18,5$ a 24,5$)$. Respecto a la variable Lugar de residencia, los hallazgos obtenidos son el $71,4 \%$ de los deportistas residen en el municipio de Bello y $28,6 \%$ residen en el municipio de Medellín (Tabla 1 ).

Tabla 1. Características sociodemográficas y antropométricas.

\begin{tabular}{|c|c|c|c|}
\hline \multicolumn{2}{|c|}{ Variable } & Frecuencia & Porcentaje (\%) \\
\hline \multirow{2}{*}{$\begin{array}{c}\text { Lugar de } \\
\text { residencia: }\end{array}$} & Bello & 10 & 71,4 \\
\cline { 2 - 4 } & Medellín & 4 & 28,6 \\
\hline Variable & Media & $\begin{array}{c}\text { Desviación } \\
\text { estándar }\end{array}$ \\
\hline Edad: & 18,29 & 1,86 \\
\hline Peso: & 65,71 & 8 \\
\hline Estatura: & 1,74 & 0,05 \\
\hline IMC: & 21,42 & 2,06 \\
\hline
\end{tabular}

Características del entrenamiento. Respecto a los antecedentes de lesiones deportivas, se encontraron lesión ligamentosa $(42,9 \%)$, lesión muscular $(21,4 \%)$, lesión ósea $(14,3 \%)$, lesión tendinosa $(7,1 \%)$ y el $14,3 \%$ de los participantes, no tenían antecedentes de lesión deportiva. Al verificar la incidencia de lesiones de los ligamentos, según la posición de juego, se evidenció que los volantes (no especificado tipo) y defensas fueron las posiciones con mayor cantidad de lesiones reportadas (con una frecuencia de 2 deportistas en cada posición), seguido por 1 arquero y 1 delantero.

La superficie de entrenamiento es una constante en los participantes, debido a que algunos días entrenan en grama sintética y otros días en grama natural, según la disponibilidad de la cancha. Al verificar el uso de estabilizadores articulares externos (rodilleras, tobilleras, vendajes, entre otros), se encontró que el $28,6 \%$ de los deportistas utilizan tobilleras y el $71,4 \%$ no utiliza ningún elemento. En relación a la posición de juego de los participantes, se encontró que 2 de ellos juegan en la posición de arquero, 5 defensas, 2 delanteros y 5 volantes. En cuanto a la dominancia de miembro inferior, se evidenció que 12 deportistas consideran más hábil su pierna derecha y 2 deportistas su pierna izquierda. La frecuencia de entrenamiento (veces por semana) permitió determinar una mediana de 3 con rango inter-cuartil de 3 y 3 . En el tiempo en la práctica deportiva, se encontró una media de 9.50\$3.99 años (Tabla 2). 
Tabla 2. Características del entrenamiento.

\begin{tabular}{|c|c|c|c|}
\hline \multicolumn{2}{|l|}{ Variable } & Frecuencia & Porcentaje (\%) \\
\hline \multirow{5}{*}{ Lesión deportiva } & Ligamentosa & 6 & 42,9 \\
\hline & Muscular & 3 & 21,4 \\
\hline & Ósea & 2 & 14,3 \\
\hline & Tendinosa & 1 & 7,1 \\
\hline & Ninguna & 2 & 14,3 \\
\hline \multirow{2}{*}{$\begin{array}{c}\text { Estabilizadores articulares } \\
\text { externos }\end{array}$} & Tobillera & 4 & 28,6 \\
\hline & Ninguna & 10 & 71,4 \\
\hline \multirow{4}{*}{ Posición de juego } & Arquero & 2 & 14,3 \\
\hline & Defensa & 5 & 35,7 \\
\hline & Delantero & 2 & 14,3 \\
\hline & Volante & 5 & 35,7 \\
\hline \multirow{2}{*}{ Dominancia } & Derecho & 12 & 85,8 \\
\hline & Izquierdo & 2 & 14,2 \\
\hline \multicolumn{2}{|l|}{ Variable } & Media & $\begin{array}{l}\text { Desviación } \\
\text { estándar }\end{array}$ \\
\hline \multicolumn{2}{|c|}{ Tiempo en la práctica deportiva } & 9,50 & 3,99 \\
\hline \multicolumn{2}{|l|}{ Variable } & Mediana & Rango intercuartil \\
\hline \multicolumn{2}{|c|}{ Frecuencia de entrenamiento (días por semana) } & 3,00 & 3-3 \\
\hline
\end{tabular}

Variables clínicas. Con el objetivo de describir la medición de la flexibilidad con el test de Bleck, en la primera toma, se analizaron los datos con frecuencias y porcentajes, tomando la variable como cualitativa. Se encontró que 13 deportistas tenían retracción moderada de isquiotibiales en el miembro inferior derecho y una (1) deportista presentó retracción severa de isquiotibiales, en el mismo miembro. Posterior a la intervención de 6 semanas, ningún deportista se clasificó con retracción severa de isquiotibiales, 6 deportistas se encontraron sin retracción, 5 con retracción moderada y 3 con retracción leve de isquiotibiales de miembro inferior derecho. Para el miembro inferior izquierdo, se evidenció que todos los participantes presentaban retracción moderada en la evaluación inicial; posterior a la aplicación del protocolo, se conoció que 7 futbolistas se encontraban sin retracción, 5 presentaron retracción leve y 2 retracción moderada. Respecto al control neuromuscular evaluado con el Star Excursion Balance Test (SEBT), se encontró que la dirección con mayor alcance en la toma inicial de la extremidad inferior derecha, corresponde al posterior, con una media de $87,86 \mathrm{~cm}$; el miembro inferior izquierdo correspondió a la dirección posterior con media de $89,64 \mathrm{~cm}$. En la toma final, la dirección de mayor alcance fue la posteromedial para ambos miembros inferiores, con medias de 
93,57 y $93,21 \mathrm{~cm}$, para el derecho y el izquierdo, respectivamente; la descripción de las distancias alcanzadas en el SEBT antes y después, se muestran en la tabla 3. Las direcciones en las que se evidenciaron mayores cambios en la evaluación final respecto a la inicial fueron en el miembro inferior derecho, los alcances posteromediales, con una diferencia de $12,21 \mathrm{~cm}$, medial, con una diferencia de $7,14 \mathrm{~cm}$ y posterolateral, con una diferencia de $5,5 \mathrm{~cm}$; en el miembro inferior izquierdo, las direcciones mediales, con una diferencia de $8,79 \mathrm{~cm}$, posteromedial con diferencia de $7,35 \mathrm{~cm}$ y posterior, con diferencia de $5,36 \mathrm{~cm}$. Considerando el riesgo de lesión, según Olmsted et al. (2002), (diferencia $>4 \mathrm{~cm}$ en la excursión anterior); un 35\% (5 deportistas) de la población presenta un balance dinámico alterado en la toma inicial y un $21 \%$ (3 deportistas) de la población presenta un balance dinámico alterado en la toma final (Tabla 3).

Tabla 3. Control neuromuscular evaluado con SEBT.

\begin{tabular}{|c|c|c|c|c|}
\hline \multirow[b]{2}{*}{$\begin{array}{l}\text { Dirección } \\
\text { sebt }\end{array}$} & \multicolumn{2}{|c|}{ Derecho } & \multicolumn{2}{|c|}{ Izquierdo } \\
\hline & $\begin{array}{l}\text { Promedio } \\
\text { inicial }\end{array}$ & $\begin{array}{l}\text { Promedio } \\
\text { final }\end{array}$ & $\begin{array}{c}\text { Promedio } \\
\text { inicial }\end{array}$ & $\begin{array}{c}\text { Promedio } \\
\text { final }\end{array}$ \\
\hline Anterior & 76,79 & 77,5 & 76,21 & 79,29 \\
\hline Antero-lateral & 71,43 & 70,36 & 69,6 & 73,57 \\
\hline Lateral & 73,57 & 78,57 & 77,14 & 79,29 \\
\hline $\begin{array}{l}\text { Postero- } \\
\text { lateral }\end{array}$ & 82,86 & 88,36 & 87,5 & 90,36 \\
\hline Posterior & 87,86 & 92,86 & 89,64 & 95 \\
\hline $\begin{array}{l}\text { Postero- } \\
\text { medial }\end{array}$ & 81,36 & 93,57 & 85,86 & 93,21 \\
\hline Medial & 81,43 & 88,57 & 81,21 & 90 \\
\hline Antero-medial & 78,93 & 78,57 & 78,57 & 80 \\
\hline
\end{tabular}

En cuanto al control neuromuscular de miembro inferior evaluado con el hop test, se pudo evidenciar que no se presentaron asimetrías importantes de un miembro inferior, con respecto al otro, exceptuando solo algunos casos; esto se puede evidenciar con las medidas obtenidas de las distancias, que fueron en promedio inicial, para miembro inferior derecho, de $458,5 \mathrm{~cm}$ y para miembro inferior izquierdo, de $480,93 \mathrm{~cm}$. Por otro lado, se pudo observar que la mayoría de participantes mostraron cambios importantes en los resultados del hop test final, donde se obtuvo una media final, para miembro inferior derecho, de $548,6 \mathrm{~cm}$ y para miembro inferior izquierdo, de 533,5cm; con excepción de un participante, que no mostró cambios en la distancia alcanzada en el hop test inicial y final para el miembro inferior derecho, donde obtuvo 592cm, en ambas mediciones (Tabla 4). 
Tabla 4. Control neuromuscular de miembros inferiores evaluado con triple hop test.

\begin{tabular}{|c|c|c|c|c|c|c|}
\hline \multirow{2}{*}{ Participante } & \multicolumn{2}{|c|}{ Promedio inicial } & \multirow{2}{*}{$\begin{array}{c}\text { Índice } \\
\text { simetría } \\
\text { inicial } \\
(\%)\end{array}$} & \multicolumn{2}{|c|}{ Promedio final } & \multirow{2}{*}{$\begin{array}{c}\text { Índice } \\
\text { simetría } \\
\text { final (\%) }\end{array}$} \\
\hline & Derecho & Izquierdo & & Derecho & Izquierdo & \\
\hline 1 & 499,6 & 503,7 & 99 & 506 & 509 & 99 \\
\hline 2 & 586 & 618 & 94 & 624 & 596 & 95 \\
\hline 3 & 502,8 & 520,6 & 96 & 502,8 & 520,6 & 96 \\
\hline 4 & 391 & 429,7 & 90 & 464 & 480,7 & 96 \\
\hline 5 & 555,9 & 536,3 & 96 & 600,7 & 581,9 & 96 \\
\hline 6 & 324,5 & 372,5 & 87 & 455 & 473,1 & 96 \\
\hline 7 & 337,3 & 348,5 & 96 & 474 & 525,7 & 90 \\
\hline 8 & 489 & 532 & 91 & 595 & 524 & 94 \\
\hline 9 & 527 & 516 & 97 & 530,5 & 519,8 & 97 \\
\hline 10 & 267 & 367,5 & 73 & 650,3 & 624,2 & 95 \\
\hline 11 & 407,5 & 406,5 & 99 & 546,9 & 566,13 & 96 \\
\hline 12 & 474,6 & 481,4 & 98 & 613 & 648,2 & 94 \\
\hline 13 & 592 & 596 & 99 & 592 & 624,5 & 94 \\
\hline 14 & 464 & 505 & 91 & 548,6 & 533,5 & 97 \\
\hline $\begin{array}{l}\text { Promedio } \\
\text { total }\end{array}$ & 458,5 & 480,93 & 93 & 550,21 & 552 & 95 \\
\hline
\end{tabular}

En las pruebas de flexibilidad, tanto Bleck como Ely, se obtuvieron diferencias estadísticamente significativas en los rangos de flexión y de extensión de rodilla, para ambos miembros inferiores, en la mayoría de los jugadores. Respecto al índice de simetría obtenido en el hop test, no se encontraron diferencias estadísticamente significativas, incluso, la variación de la mediana entre ambas tomas solo fue del 1\%; sin embargo, al analizar los datos individuales de esta prueba, se evidencia un incremento en la distancia obtenida en la segunda toma con respecto a la primera, en la mayoría de los jugadores (Tabla 5).

Tabla 5. Resultados de flexibilidad evaluado con test de Bleck y test de Ely, control neuromuscular evaluado con SEBT y triple hop test.

\begin{tabular}{|c|c|c|c|c|c|}
\hline \multicolumn{2}{|c|}{ Variable } & Inicial & Final & Estadístico & $\mathbf{P}$ \\
\hline \multicolumn{2}{|l|}{ Test de Bleck derecho } & $136(131-141)$ & $160(151-162)$ & $-3,298$ & $0,001^{w}$ \\
\hline \multicolumn{2}{|c|}{ Test de Bleck izquierdo } & $136(132-141)$ & $161(158-168)$ & $-3,109$ & $0,002^{W}$ \\
\hline \multicolumn{2}{|l|}{ Test de Ely derecho } & $10,36 \pm 4,87$ & $7,89 \pm 5,82$ & 1,607 & $0,132^{\top}$ \\
\hline \multicolumn{2}{|l|}{ Test de Ely izquierdo } & $13,32 \pm 6,13$ & $8 \pm 4,13$ & 4,489 & $0,001^{\top}$ \\
\hline \multicolumn{2}{|c|}{ Índice de simetría con triple hop test } & $95(90-98)$ & $96(94-96)$ & $-0,920$ & $0,357^{\mathrm{W}}$ \\
\hline \multirow{2}{*}{ SEBT anterior } & Derecha & $80(70-80)$ & $80(70-80)$ & $-0,378$ & $0,705^{\mathrm{W}}$ \\
\hline & Izquierda & $80(70-80)$ & $80(70-82)$ & $-1,684$ & $0,092^{W}$ \\
\hline \multirow{2}{*}{ SEBT anterolateral } & Derecha & $70(67-80)$ & $70(67-76)$ & $-0,723$ & $0,47^{\mathrm{W}}$ \\
\hline & Izquierda & $70(60-80)$ & $75(70-80)$ & $-1,674$ & $0,094^{W}$ \\
\hline \multirow{2}{*}{ SEBT lateral } & Derecha & $73,57 \pm 9,88$ & $78,57 \pm 10,27$ & $-1,803$ & $0,095^{\top}$ \\
\hline & Izquierda & $80(70-82)$ & $80(70-90)$ & $-1,000$ & $0,317^{\mathrm{W}}$ \\
\hline \multirow{2}{*}{ SEBT posterolateral } & Derecha & $80(80-90)$ & $90(87-90)$ & $-2,275$ & $0,023^{W}$ \\
\hline & Izquierda & $90(87-90)$ & $90(83-100)$ & $-1,224$ & $0,221^{\mathrm{W}}$ \\
\hline \multirow{2}{*}{ SEBT posterior } & Derecha & $87,86 \pm 11,044$ & $92,86 \pm 9,13$ & $-2,385$ & $0,033^{\top}$ \\
\hline & Izquierda & $90(80-96)$ & $100(90-100)$ & $-1,951$ & $0,051^{\mathrm{W}}$ \\
\hline \multirow{2}{*}{ SEBT posteromedial } & Derecha & $90(80-93)$ & $90(90-100)$ & $-2,192$ & $0,028^{W}$ \\
\hline & Izquierda & $86(80-90)$ & $90(90-100)$ & $-2,716$ & $0,007^{\mathrm{W}}$ \\
\hline \multirow{2}{*}{ SEBT medial } & Derecha & $80(70-90)$ & $90(80-92)$ & $-2,640$ & $0,008^{W}$ \\
\hline & Izquierda & $80(80-90)$ & $90(80-100)$ & $-2,410$ & $0,016^{W}$ \\
\hline \multirow{2}{*}{ SEBT anteromedial } & Derecha & $80(70-82)$ & $80(70-82)$ & $-0,302$ & $0,763^{\mathrm{W}}$ \\
\hline & Izquierda & $80(73-80)$ & $80(70-90)$ & $-2,410$ & $0,016^{\mathrm{W}}$ \\
\hline
\end{tabular}

* T. T- Student, presentado con media y desviación estándar.

* W. Wilcoxon, presentado con mediana y rango intercuartil. 
Adicionalmente, se analizó la posición de juego de los participantes con los resultados obtenidos en el hop test, tanto en la evaluación inicial como en la evaluación final, encontrando diferencias estadísticamente no significativas $(P>0,05)$; no obstante, se evidenciaron cambios en promedio de las mediciones inicial y final de los participantes, principalmente, de los defensas, con un cambio de $122 \mathrm{~cm}$, en el miembro inferior derecho y $91 \mathrm{~cm}$, en el miembro inferior izquierdo (Tabla 6).

Tabla 6. Resultado triple hop test según la posición de juego.

\begin{tabular}{|c|c|c|c|c|c|c|c|c|c|c|}
\hline \multirow{2}{*}{$\begin{array}{l}\text { Posición } \\
\text { de juego }\end{array}$} & \multicolumn{5}{|c|}{ Pierna derecha } & \multicolumn{5}{|c|}{ Pierna izquierda } \\
\hline & Inicial & Final & Diferencia & $\mathbf{P}$ & Total & Inicial & Final & Diferencia & $\mathbf{P}$ & Total \\
\hline Arquero & $440 \pm 69,29$ & $530 \pm 92,63$ & 89,5 & 0,12 & \multirow{4}{*}{0,098} & $481 \pm 72,12$ & $503 \pm 30,4$ & 21,5 & 0,6 & \multirow{4}{*}{0,082} \\
\hline Defensa & $447,2 \pm 103,69$ & $569 \pm 60,77$ & 121,8 & 0,16 & & $478,2 \pm 63,50$ & $569 \pm 61,63$ & 91,2 & 0,15 & \\
\hline Delantero & $497 \pm 125,87$ & $586 \pm 54,44$ & 88,5 & 0,33 & & $512 \pm 149,90$ & $581 \pm 21,21$ & 69 & 0,59 & \\
\hline Volante & $461,8 \pm 124,35$ & $526 \pm 67,31$ & 63,8 & 0,1 & & $471 \pm 107,08$ & $543 \pm 60,05$ & 71,8 & 0,08 & \\
\hline
\end{tabular}

*Valor inicial, final y diferencia reportada en centímetros.

**DE. Desviación estándar.

\section{DISCUSIÓN}

De acuerdo con los resultados obtenidos en el estudio, se logró describir las características sociodemográficas, antropométricas y de entrenamiento de los futbolistas que hicieron parte del estudio; además, se determinó el estado inicial del control neuromuscular y flexibilidad en miembros inferiores, previo a la aplicación de un entrenamiento pliométricoyseidentificaronloscambiosdelaspruebas aplicadas, posterior a la realización del protocolo.

Para realizar la caracterización antropométrica en la

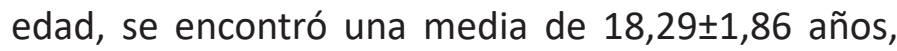
contrastado con la literatura, que reporta estudios, como el realizado por Pérez et al. (2017) y Ospina et al. (2014), donde en el 2016, se encontraron datos similares, con medias de $21,2 \pm 3,1$ y en el 2014 , en un estudio realizado con futbolistas profesionales, encontraron medias de edad en dos grupos de $25,8 \pm 4,3$ y $24,1 \pm 3,8$. El peso tuvo como resultado una media de 65,71 kilogramos, con \pm 8 ; se evidencia un peso más bajo respecto a estudios realizados en Madrid, España, en el 2016 y 2015, con medias de $67,93 \pm 6,33$ y $69,2 \pm 1$, respectivamente $(8,6)$; igual sucede, comparado con un estudio con población de características similares, pero de mayor edad, realizado por Ospina et al. (2014), con futbolistas profesionales, donde se hallaron pesos en dos grupos, con medias de 75,6kg $\pm 5,7$ y 78,6 kg $\pm 5,2$. Por otro lado, en la estatura, se encontró una media de 1,74 metros $\pm 2,06$, una talla más baja que la reportada en estudios realizados por Pangrazio \& Forriol (2016), Di Paco et al. (2017) y Búa et al. (2013), donde se encontraron datos de 1,76 \pm 0.06 ; $1,82 \pm 0.07$ y $1,75 \pm 0,075$, respectivamente $(8,10,9)$. El IMC, se concretó en un valor que se ubica dentro del rango normal, dado por la OMS, con una media de $21,42 \pm 2,06$, datos similares a los obtenidos en el 2016 ,con media de $21,88 \pm 1,49$, pero mucho menor a los datos obtenidos por Ospina et al. (2014), Di Paco et al. (2017) y Márquez et al. (2019), donde se registraron medias de $24,9 \pm 1,7,24,5 \pm 1$ y $23,6 \pm 1,3$, respectivamente; el IMC más bajo reportado en jugadores de características similares, se encontró en Argentina, con un valor de 19,2 $\pm 1,6$, en un estudio realizado por Búa et al. (2013).

Con relación a las características del entrenamiento, se indagó sobre los antecedentes deportivos de miembro inferior y se encontró que, según el tipo de lesión, la lesión ligamentosa es la de mayor frecuencia $(42,9 \%)$, información concordante con los resultados obtenidos en un estudio realizado en la Universidad de Antioquia de Medellín, Colombia, donde se siguió, prospectivamente durante 1 año, a 24 jugadores de la primera categoría al equipo de fútbol Equidad Seguros, para determinar las características de lesiones derivadas a la práctica deportiva, encontrando que la mayoría de las lesiones fueron ligamentosas, seguidas de las musculares, con un porcentaje del 25\%, para esguince de ligamento fibulotalar anterior y 16,6\%, para lesión del ligamento cruzado anterior (Márquez et al. 2015). 
Algo similar, se constató en otro estudio realizado en Suiza, por Read et al. (2016), con jugadores de fútbol masculinos, donde la incidencia de lesiones ligamentosas fue del $83 \%$, siendo el segmento más comprometido la articulación de la rodilla; sin embargo, para el presente estudio, no se indagó por la región o segmento comprometido, limitando los análisis asociados; en contraste con otro informe bibliográfico realizado por Márquez et al. (2019), reporta antecedentes de lesión en el 35\% de la población, principalmente de tipo muscular, como desgarro muscular de isquiotibiales, cuádriceps y aductores (Márquez et al. 2019). Además, se encontraron diferencias en otro estudio publicado por Watson et al. (2019), en donde las distensiones y los desgarros musculares son las lesiones más comunes, pero la fractura se presenta con mayor frecuencia que las lesiones en tejidos blandos, las contusiones y las laceraciones; caso contrario, ocurre en un estudio transversal reportado en España, por Pangrazio \& Forriol (2016), donde la contusión es la lesión más frecuente, seguida por la distensión o desgarro muscularyubicandoconmenorfrecuencia, laslesiones ligamentosas, como esguinces o ruptura del LCA.

En adición a lo anterior y respecto a la dominancia de los jugadores, se encontró que la mayoría de los jugadores $(85,8 \%)$ tienen dominancia derecha, similar a lo encontrado en los reportes de Márquez et al. (2019), donde el porcentaje fue $72,5 \%$ y en 2016 , donde el porcentaje de la población con dominancia derecha fue 65,2\% (Pangrazio \& Forriol, 2016).

Respecto al tipo de calzado utilizado, todos los participantes reportaron que usan guayos y tenisguayos, según la superficie donde van a jugar. Se encontró un estudio que incluyó 24 futbolistas sanos y utilizaron la prueba BESS (Balance Error Scoring System), para medir la inestabilidad en 6 condiciones, donde se encontró que el uso de los tres modelos de zapatos (botas de fútbol, zapatos de futsal y zapatos para correr), dieron mayor estabilidad, que cuando el sujeto estaba descalzo. Solo en la superficie firme las botas de fútbol eran estadísticamente mejores que los de futsal (Notarnicola et al. 2015).

En cuanto a la superficie de entrenamiento, Watson et al. (2019) descubrieron que el entrenamiento en grama natural se asoció con un riesgo 2,8 veces mayor de lesión en extremidades inferiores, mientras que O'Kane et al. (2016) encontraron que las lesiones en los juegos de fútbol juvenil no variaron significativamente, cuando se comparó la grama artificial con la grama natural; para el presente estudio, los participantes entrenan en ambas superficies según las características del entrenamiento y disponibilidad del escenario, por lo que no fue posible sacar conclusiones del impacto de la superficie de juego.

Referente a la flexibilidad, un estudio publicado por Cejudo et al. (2014), donde compara los valores de flexibilidad con respecto a la posición de juego, en el cual, se obtiene un promedio mayor de flexibilidad de isquiotibiales en los arqueros con respecto a los demás jugadores de campo, a pesar de no tener una muestra significativa, los resultados son similares al presente estudio, al comparar arqueros versus los jugadores de campo.

En un estudio diferente realizado por Cejudo et al. (2019) encontraron, al realizar la medición de flexibilidad de miembros inferiores en diferentes categorías de futbolistas entre 8 y 19 años de edad, que los rangos de movilidad fueron mayores en las categorías más jóvenes y, por el contrario, existía una disminución progresiva de estos en los equipos de mayor edad. Por otro lado, Daneshjoo et al. (2013), donde evaluaron la flexibilidad de la cadera, mediante la flexión máxima de cadera con la rodilla en extensión, se registró que hubo diferencias significativas entre la flexibilidad de la pierna dominante versus la pierna no dominante.

En estudios realizados en Nueva Zelanda y España (2017), se usaron pruebas como single leg hop test y SEBT, para determinar el nivel de control neuromuscular en futbolistas jóvenes, pruebas utilizadas en este estudio, para los mismos fines. En la evaluación del control neuromuscular realizada mediante el hop test, se pudo evidenciar que los participantes del presente estudio no mostraron asimetrías mayores del $15 \%$, entre ambos miembros inferiores; en su mayoría, solo un participante tuvo una diferencia mayor a este porcentaje en los índices de simetría; sin embargo, un estudio realizado por Read et al. (2019) muestra que las asimetrías entre miembros inferiores en atletas jóvenes oscilan entre el 15 y el $20 \%$ y subraya que existe una mayor variabilidad del movimiento en poblaciones jóvenes, especialmente, entre 14 a 25 años de edad. Por otro lado, Meierbachtol et al. (2017) realizaron un estudio para observar las mejoras cuantitativas en los resultados del hop test en 71 participantes después de un programa de entrenamiento neuromuscular 
durante 6 semanas, encontrando una diferencia en el índice de simetría pre-test, de $87 \%$ y post-test, de $92 \%$, evidenciando un cambio relevante, datos que concuerdan con los datos obtenidos en este estudio.

Al analizar la pruebaSEBT, se evidencia que la dirección con mayor alcance en la toma inicial corresponde a posteromedial, con 87,86 y $89,64 \mathrm{~cm}$, para miembro inferior derecho e izquierdo, respectivamente, lo cual, difiere levemente con estudios similares realizados por la facultad de fisioterapia de la UNAL de Bogotá (Mancera et al. 2013), donde las direcciones con mayor alcance fueron media, con $88,4 \mathrm{~cm}$ y posteromedial, con $91,9 \mathrm{~cm}$ (promedio) y el colegio de Kinesiólogos de Chile (Núñez et al. 2011), obtuvo mayor alcance en la dirección posteromedial, con $115 \mathrm{~cm}$, para ambos miembros inferiores. Estudios adicionales, como el realizado por el grupo de investigación PROFITH de Granada (Molina et al. 2018), adoptan la versión modificada del SEBT también llamada "Y balance test", en la cual, solo se analizan 3 direcciones (anterior, posteromedial y posterolateral), donde reportaron solo la distancia anterior de la pierna izquierda, con una media total de $81,4 \mathrm{~cm}$, para jugadores lesionados, un promedio de $82,3 \mathrm{~cm}$ y un promedio de $79,74 \mathrm{~cm}$, para no lesionados, predice el número de esguinces de tobillo, para esa misma pierna, después de haber aplicado el test a los dos grupos; para la pierna derecha, la media total fue de $80,6 \mathrm{~cm}, 81,1 \mathrm{~cm}$, en jugadores lesionados y $79,6 \mathrm{~cm}$ en no lesionados.

Tras la aplicación de un entrenamiento pliométrico, se pueden encontrar mejorías significativas en el control neuromuscular, medido mediante el Hop test, resultados que concuerdan con el estudio realizado por Ramírez-Campillo et al. (2015), donde tras la aplicación de un entrenamiento pliométrico unilateral y bilateral, se encuentran efectos significativos en el equilibrio, además de una mejoría en el desarrollo de actividades explosivas, como en los saltos, posiblemente, inducidas por adaptaciones neuromusculares, que no fueron medidas de manera específica. Asimismo, Wang \& Zhang (2016) encontraron que la aplicación de un entrenamiento pliométrico aplicado a futbolistas, debidamente dirigido y supervisado, tienen influencias directas sobre habilidades particulares de los jugadores de fútbol y capacidad de salto vertical en individuos de cualquier edad; además, las mejoras incluyen el fortalecimiento muscular y tendinoso, lo que resulta en la capacidad de evitar lesiones.
Por otro lado, Hammami et al. (2019), al comparar los efectos entre un entrenamiento de fuerza de contraste y un entrenamiento pliométrico, hallando que ambos entrenamientos tienen efectos positivos en la mejora del salto en cuclillas, salto en contramovimiento, realizar cambios de dirección y de adaptaciones neuromusculares de miembros inferiores, sin diferencias significativas entre ambos grupos. Por su parte, Chang \& Wang (2018) estudiaron el efecto de un entrenamiento pliométrico en la activación neuromuscular de los miembros inferiores, encontraron mejoría en la altura de los saltos y distancia de los mismos, resultado concordante con lo registrado el presente estudio. En cuanto al estudio adelantado por McPhail (2017), se encontró, también, que el entrenamiento pliométrico ofrece mejoría en la altura del salto de caída y el salto en contra-movimiento, que es similar a lo hallado en un artículo realizado en Bogotá, Colombia, por Luis Alberto Cardozo, quien compara la diferencia del salto vertical en dos grupos, realizando una evaluación antes y después de una intervención de Entrenamiento pliométrico $(\mathrm{PL})$ y Entrenamiento con Resistencias elásticas (TE), durante 12 semanas a cada grupo; al finalizar el estudio, se encontró ganancia en la capacidad de salto en el grupo PL, del $18,4 \%$, comparado con la evaluación inicial, a diferencia del grupo TE, el cual, solo tuvo un aumento de 10,7\% (Cardozo \& Yáñez, 2017).

Aunque al inicio, se explicó a los deportistas que no debían realizar entrenamientos por fuera de los programados, es posible que los cambios identificados en este estudio no correspondan exclusivamente a la intervención, sino que, también, se puedan deber a entrenamientos adicionales practicados por los deportistas durante el periodo de tiempo, en el que transcurrió el estudio.

Se presentó pérdida de participantes, en el proceso de recolección de información, disminuyendo la muestra de 31 a 14 participantes, debido a que, para ser incluidos en los análisis finales, cada sujeto tenía que completar, al menos, el $80 \%$ de todas las sesiones de intervención, es decir, 9 de 12 sesiones y participar en ambas evaluaciones, tanto inicial como la final.

El tamaño de la muestra no permite extrapolar los resultados a poblaciones con características similares, además disminuye la posibilidad de realizar comparación de un grupo control versus grupo intervención; sin embargo, puede ser un punto de 
partida para la realización de nuevas investigaciones.

No se indagó por el segmento comprometido al verificar los antecedentes de lesión, lo que limitó la realización de los análisis respectivos y comparación con otros estudios que sí lo reportan.

\section{CONCLUSIONES Y RECOMENDACIONES}

El fútbol es uno de los deportes donde se presenta mayor índice de lesiones de miembro inferior en quienes lo practican. Dichas lesiones pueden estar asociadas a múltiples factores, dentro de los cuales, se pueden destacar el déficit de control neuromuscular y la flexibilidad; por ello, se hace fundamental la búsqueda de estrategias de intervención que apunten a lograr la mejoría de factores alterados, que pueden conllevar a los jugadores a sufrir una lesión, ya sea durante el entrenamiento o durante el juego competitivo. Igualmente, proporcionar información acerca de estos factores a los deportistas y al equipo interdisciplinario es útil para identificar los posibles riesgos y prevenir lesiones deportivas asociadas.

Se concluye que, tras la aplicación de un protocolo de entrenamiento pliométrico adicional al convencional para los futbolistas, se pueden obtener cambios positivos en la flexibilidad en los miembros inferiores, específicamente, en la musculatura, que interviene en la flexión y la extensión de rodilla. Asimismo, al conocer las implicaciones neuromusculares requeridas para la realización de la actividad deportiva y la prevención de lesiones asociadas a ello, se resalta la importancia de la inclusión del entrenamiento pliométrico, porque logra la activación óptima de todos los componentes neuromusculares, disminuyendo desbalances presentes en los miembros inferiores que predisponen a alguna lesión.

Este tipo de estudios pueden contribuir a resaltar la importancia del papel del fisioterapeuta en dichos escenarios $y$, especialmente, en la implementación de protocolos estructurados, que apunten a la prevención de lesiones y no solo en procesos de rehabilitación de las mismas.

Para estudios posteriores, se sugiere una muestra más amplia que facilite obtener datos más confiables al ser analizados estadísticamente y permita extrapolar los resultados a poblaciones con características semejantes. Este tipo de estudios pueden incluir, también, comparaciones entre diferentes equipos de fútbol, jugadores de diferente categoría y ambos sexos e, incluir, otro tipo de deporte, con componente acíclico.

\section{REFERENCIAS}

1. BÚA, N.; RODRÍGUEZ, A.; GARCÍA, G. 2013. Perfil funcional y morfológico en jugadores de fútbol amateur de Mendoza, Argentina. Apunt Med l'Esport. 48(179):89-96. https://doi.org/10.1016/j.apunts.2012.07.001

2. CARDONA, O.; OSORIO, M. 2008. Entrenamiento neuromuscular para la prevención de lesiones de rodilla en femeninas adolescentes. Universidad Tecnológica de Pereira (Pereira).

3. CARDOZO, L.; YÁÑEZ, C. 2017. Efecto del entrenamiento pliométrico vs. thera-band en la altura de salto vertical en jóvenes futbolistas. Journal of Sport and Health Research. 9(2):247-262.

4. CEJUDO, A.; ROBLES, F.; AYALA, F.; CROIX, M.; ORTEGA, E.; SANTONJA, F.; SAINZ, P. 2019. Age-related differences in flexibility in soccer players 8-19 years old. PeerJ. 7:e6236. https://doi.org/10.7717/peerj.6236

5. CEJUDO, A.; SAINZ DE BARANDA, P.; AYALA, F.; SANTOJA, F. 2014. Perfil de flexibilidad de la extremidad inferior en jugadores de fútbol sala. Revista Internacional de Medicina y Ciencias de la Actividad Física y el Deporte. 14(55):509-525.

6. CHANG, H.; WANG, C. 2018. The Effect of 8-Week Plyometric Training on Neuromuscular Activation and Sports Performance for PrePubertal Boys. Asian J Coach Sci. 2(1):12-24. https://doi.org/10.29426/ajcs.201812_2(1).0002

7. DANESHJOO, A.; RAHNAMA, N.; MOKHTAR, A.; YUSOFT, A. 2013. Bilateral and Unilateral Asymmetries of Isokinetic Strength and Flexibility in Male Young Professional Soccer Players. J Hum Kinet. 36:45-53. https://doi.org/10.2478/hukin-2013-0005

8. DI PACO, A.; DUBÉ, B.; LAVENEZIANA, P. 2017. Cambios en la respuesta ventilatoria al esfuerzo en deportistas entrenados: efectos beneficiosos sobre la fisiología respiratoria más allá del rendimiento cardiovascular. Arch Bronconeumol 53(5):237-244.

http://dx.doi.org/10.1016/j.arbres.2016.11.023 
9. HAMMAMI, M.; GAAMOURI, N.; SHEPHARD, R.; CHELLY, M.S. 2019. Effects of contrast strength vs. plyometric training on lowerlimb explosive performance, ability to change direction and neuromuscular adaptation in soccer players. J Strength Cond Res. 33(8):2094-2103.

https://doi.org/10.1519/jsc.0000000000002425

10. HAWKINS, R.; FULLER, C. 1999. Prospective epidemiological study of injuries in four English professional football clubs. British Journal of Sports Medicine. 33(3):196-203. https://dx.doi.org/10.1136\%2Fbjsm.33.3.196

11. KUNZ, M. 2006. Gran Censo FIFA. FIFA Magazine.

12. LEPHART, S.; PINCIVERO, D.; ROZZI, S. 1998. Proprioception of the ankle and knee. Sports Medicine. 25(3):149-155.

https://doi.org/10.2165/00007256-19982503000002

13. LLANA, S.; PÉREZ, P.; LLEDÓ, E. 2010. La epidemiologia en el fútbol: una revisión sistemática. Revista Internacional de la Medicina y Ciencias de la Actividad Física y del deporte. 10(37):22-40.

14. MANCERA, E.; HERNÁNDEZ, E.; HERNÁNDEZ, F.; PRIETO, L.; QUIROGA, L. 2013. Efecto de un programa de entrenamiento físico basado en la secuencia de desarrollo sobre el balance postural en futbolistas: Ensayo controlado aleatorizado. Revista de la Facultad de Medicina. 61(4):339-347.

15. MÁRQUEZ, J.; SUÁREZ, G.; QUICENO, C. 2015. Lesiones en futbolistas de un equipo sudamericano durante 1 año de seguimiento. Revista Cubana de Ortopedia y Traumatología. 29(1):65-75.

16. MÁRQUEZ, W.; GÓMEZ, J.; GALLO, J.; ESPINOSA, B.; RIVAS, N.; LLANO, J.; OSORIO, J.; MARTIN, H.D. 2019. Prevalence of labrum and articular cartilage injuries of the hip on $3 T$ magnetic resonance imaging of asymptomatic elite soccer players. Rev Esp Cir Ortop Traumatol. 63(2):77-85.

https://doi.org/10.1016/j.recot.2018.10.008

17. MCPHAIL, J. 2017. Effects of combining repeated sprints and plyometric training on repeated sprint ability in male youth soccer players. Master's Thesis Science of Sport Coaching and Fitness Testing Faculty of Sport and Health Sciences University of Jyväskylä. 1-49.

18. MEIERBACHTOL, A.; ROHMAN, E.; PAUR, E.; BOTTOMS, J.; TOMPKINS, M. 2017. Quantitative Improvements in Hop Test Scores After a 6-Week Neuromuscular Training Program. Sports Health. 9(1):22-9. https://doi.org/10.1177/1941738116667933

19. MOLINA, P.; MORCILLO, J.; CERVERA, F. 2018. Estrategias de prevención de lesiones deportivas en jóvenes futbolistas estabilidad del core y propiocepción. Rev Andal Med Deporte. 11(4):210-214.

https://doi.org/10.33155/j.ramd.2017.05.002

20. NOTARNICOLA, A.; MACCAGNANO, G.; PESCE, V.; TAFURI, S.; MERCADANTE, M.; FIORE, A.; MORETTI, B. 2015. Effect of different types of shoes on balance among soccer players. Muscles, Ligaments and Tendons Journal. 5(3):208-213. https://dx.doi. org/10.11138\%2Fmltj\%2F2015.5.3.208

21. NÚÑEZ, D.; MERINO, C.; LEPPE, J. 2011. Balance Dinámico utilizando el Star Excursion Balance Test en seleccionados nacionales de Atletismo, Básquetbol y Vóleibol. Revista oficial del colegio de kinesiólogos de Chile. 30(1):18-24.

22. O'KANE, J.; GRAY, K.; LEVY, M.; NERADILEK, M.; TENCER, A.; POLISSAR, N.; SCHIFF, M. 2016. Shoe and field surface risk factors for acute lower extremity injuries among female youth soccer players. Clin J Sport Med. 26(3):245-250. https://dx.doi. org/10.1097\%2FJSM.0000000000000236

23. OLMSTED, L.C.; CARCIA C.R.; HERTEL, J.; SHULTZ, S.J. 2002. Efficacy of the star excursion balance test in detecting reach deficits in subjects with chronic ankle instability. J Athl Train. 37(4):501-506. https://doi.org/10.1002/pri.1589

24. OSPINA, C.; GALLO, J.; AGUIRRE, D.; BALPARDA, J.; ARISTIZÁBAL, J.; OCAMPO, J. 2014. Diferencias en la respuesta hemodinámica y autonómica al estrés simpático entre futbolistas profesionales potentes y resistentes: Un estudio transversal. Apunt Med l'Esport. 49(184):113-122.

http://dx.doi.org/10.1016/j.apunts.2014.06.004 
25. PANGRAZIO, O.; FORRIOL, F. 2016. Epidemiología de las lesiones sufridas por los jugadores durante el XVI Campeonato Sudamericano Sub-17 de Fútbol. Rev Esp Cir Ortop Traumatol. 60(3):192-199.

http://dx.doi.org/10.1016/j.recot.2015.12.002.

26. PÉREZ, E.; TORRES, M.; GUTIÉRREZ, C. 2017. Prevalencia de dolor de tobillo en futbolistas de competición: estudio piloto transversal. Fisioterapia. 39(1):25-32. http://dx.doi.org/10.1016/j.ft.2016.01.001

27. RAMÍREZ-CAMPILLO, R.; BURGOS, C.; HENRIQUEZ-OLGUÍN, C.; ANDRADE, D.; MARTÍNEZ, C.; ÁLVAREZ, C.; CASTROSEPÚLVEDA, M.; MARQUES, M.; IZQUIERDO, M. 2015. Effect of unilateral, bilateral, and combined plyometric training on explosive and endurance performance of young soccer players. Natl Strength Cond Assoc. 29(5):1317-1328.

https://doi.org/10.1519/jsc.0000000000000762

28. READ, P.; OLIVER, J.; DE STE CROIX, M.; MYER, G.; LLOYD, R. 2016. Neuromuscular Risk Factors for Knee and Ankle Ligament Injuries in Male Youth Soccer Players. Sports Med. 46(8):1059-1066.

https://doi.org/10.1007/s40279-016-0479-z

29. READ, P.; OLIVER, J.; DE STE CROIX, M.B.; MYER, G.; LLOYD, R. 2019. Review of field-based assessments of neuromuscular control and their utility in male youth soccer players. Journal of Strength and Conditioning Research. 33(1):283-299. https://dx.doi. org/10.1519\%2FJSC.0000000000002069

30. WANG, Y.; ZHANG, N. 2016. Effects of plyometric training on soccer players. Exp Ther Med. 12(2):550-554.

https://doi.org/10.3892/etm.2016.3419

31. WATSON, A.; MJAANES, J.; COUNCIL ON SPORTS MEDICINE AND FITNESS. 2019. Soccer Injuries in Children and Adolescents. Pediatrics. 144(5):e20192759.

https://doi.org/10.1542/peds.2019-2759 